\title{
On the semi-rigidity of dovetail joint for the joinery of LVL panels
}

\author{
Stéphane Roche ${ }^{1} \cdot$ Christopher Robeller $^{1} \cdot$ Laurent Humbert $^{2} \cdot$ Yves Weinand $^{1}$
}

Received: 15 September 2014/Published online: 6 June 2015

(C) Springer-Verlag Berlin Heidelberg 2015

\begin{abstract}
This paper focuses on multiple tab-and-slot joints (MTSJ) for load-carrying assembly of structural wood elements, which are inspired by traditional cabinetmaking joints and adapted for automatic fabrication and engineered wood panels such as laminated veneer lumber. First a numerical method is presented for the estimation of the connections' semi-rigid properties based on box beam samples. In a simplified model, the mechanical behavior of the joint is represented by potential elastic slips at interfaces. They are handled by the model with the help of rigidity modulus, which is determined by matching simulated and experimental deflections. Finally, the influence of tab length and contact face angle on the semi-rigidity of the joint is discussed considering a dovetail geometry of the MTSJ. Glued and screwed samples serve as references in this study.
\end{abstract}

\section{Introduction}

Multiply cross-laminated wood panels such as laminated veneer lumber (LVL) have received increasing usage in engineering structures over the last decades. These panels are particularly suitable for the design and fabrication of lightweight structural components, such as roof and floor

Stéphane Roche

stephane.roche@epfl.ch

1 Laboratory of Wood Construction, Ecole Polytechnique Fédérale de Lausanne, GC H2 711, Station 18, 1015 Lausanne, Switzerland

2 Laboratory for Computer-Aided Design and Production, Ecole Polytechnique Fédérale de Lausanne, ME A1 392, Station 9, 1015 Lausanne, Switzerland box elements, webbed beams or folded-plate structures as presented by Robeller et al. (2014). The construction of such components requires several panels to be jointed along their edges, where the connections between panels play a significant role in the load-carrying capacity of these structural elements.

State-of-the-art connections include adhesives, which provide a high stiffness of the connection, but the jointing must be done off-site, applying a defined, distributed pressure for a certain amount of time. Alternatively, metal fasteners such as screws can be used for the edge-jointing; however a large amount of screws will be necessary to achieve a sufficient stiffness of the connection. The direct application of metal fasteners to the edge of panels is not possible on thin LVL panels (ETA-11/0190 2013). Furthermore, for both screws and adhesive joints, a precise and fast alignment of parts may be difficult, especially for nonorthogonal edge connections.

The multiple tab-and-slot joints (MTSJ) applied in this paper is re-considering the advantages of traditional connections from cabinetmaking (Graubner 1987) while taking advantage of the efficiency and precision of computer numerical control (CNC) fabrication technology. It demonstrates an alternative technique, especially for the jointing of thin panels which cannot be connected with screws (EN 1995 2004), and allows for a fast and precise assembly of structural components. The final alignment of parts, the angles in-between parts and the direction of their assembly can be pre-defined through the geometry of the joint. A more detailed description of the joint is given by Robeller et al. (2014) within a folded-plate prototype, digitally fabricated and experimentally tested.

However, when designing new types of timber structures, stiffness remains one of the most important structural parameters that may be affected by the performance of the 
connection. Therefore, it is important to provide quantitative estimation of the joint degree of rigidity through wellcontrolled tests and analyses with reasonable generality and simplicity. While several previous studies (Engström 1999; Simek and Sebera 2010) have examined the mechanical behavior of conventional connections, the present work presents an analysis of CNC fabricated MTSJ in a case-study (LVL box beams with uniform cross section). For introducing the assumptions of semi-rigidity, the geometry of the joint is simply defined as a dovetail-joint (DJ) type similar to furniture connections in woodworking. Then, the MTSJ is longitudinally loaded during a threepoint bending test.

\section{Numerical model}

In this section, a one-dimensional simplified method is applied for the analysis of multi-layered elastic beams which permits reversible horizontal slip between the layers without vertical separation. Friction and irreversible gaps between successive layers are not addressed. This method, initially referred to as Gamma method, originates from the pioneer work of Möhler (1956) for up to three-layered composite systems subjected to cosine/sine load functions. Möhler's analysis was extended by Schelling (1968) to laminated composite beams made of an arbitrary number of layers and more complex loading cases such as piecewise, uniformly distributed loads of practical interest. Recent applications and developments of the method are proposed in the references Kreuzinger (1995), Natterer et al. (2004) among others. Besides these works, analytical solutions were also proposed at the beginning of the fifties by Newmark et al. (1951) for two-layered semi-rigid systems and extended by Goodman and Popov (1968) to threelayered (timber) composite beams of rectangular and equal cross-sections. Recent developments on the subject can be found by instance in Krawczyk et al. (2007) and Sousa and Da Silva (2010).

Practical and representative solutions may easily be derived for simply supported composite beams with arbitrary cross-sections and load distributions under the following basic assumptions: (1) the materials and interfaces behave linearly, (2) plane sections remain plane inside each connected element and (3) the same rotation $\theta$ and vertical displacement $w$ of the centroidal axis is considered for every element assuming that the slope of the deflection curve is small.

The boxed beam is viewed here as a three-layered beam that lies in the $x, z$ plane, with two slipping interfaces.

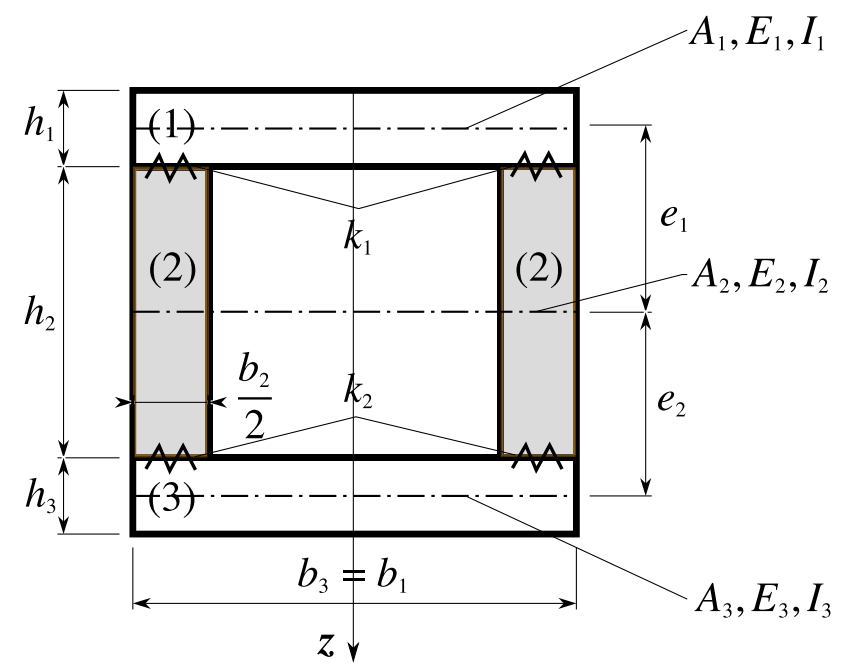

Fig. 1 Box beam cross section with semi-rigid interfaces

Relevant cross-sectional properties as well as the layer numbering are defined in Fig. 1, where the transverse coordinate $z$ is taken positive downward from the upper face. For each beam element $i$ of cross sectional area $A_{i}$ and vertical position of the reference (centroidal) axis $z_{i}$, the centroidal moment of inertia of the element cross section is denoted $I_{i}=\int_{A_{i}}\left(z-z_{i}\right)^{2} d A_{i}=\frac{1}{12} b_{i} h_{i}{ }^{3}$. The distance between the reference (centroidal) axis of the first and second elements and second and third elements is given by $e_{1}=\left(h_{1}+h_{2}\right) / 2, e_{2}=\left(h_{2}+h_{3}\right) / 2$, respectively. $E_{i}$ and $G_{i}$ are the elastic and shear modulus of the element $i$.

The two slipping interfaces are associated with the rigidity modulus $k_{1}$ and $k_{2}$ (see Fig. 1) that are kept constant along the beam length. Consequently, it is admitted that every dovetail joint behaves in a similar manner along the interface.

Denoting $\theta$ the rotation of the beam element sections, the displacement field for the beam is expressed by

$$
\begin{aligned}
& u_{i}(x, z)=u_{i}(x)-\left(z-z_{i}\right) \theta(x) \\
& w_{i}(x, z)=w(x)
\end{aligned}
$$

where $w$ is the transverse displacement. The functions $u_{i}(x)$ represent the horizontal displacements of the reference axis of each layer.

The axial strain $\varepsilon_{i}$ and corresponding stress $\sigma_{i}$ for each layer are given by

$$
\begin{aligned}
& \varepsilon_{i}=\frac{d u_{i}}{d x}-\left(z-z_{i}\right) \frac{d \theta}{d x} \\
& \sigma_{i}=E_{i} \varepsilon_{i}=E_{i}\left(\frac{d u_{i}}{d x}-\left(z-z_{i}\right) \frac{d \theta}{d x}\right)
\end{aligned}
$$


At the elements centroids, when $z=z_{i}$, one has $\sigma_{i}=E_{i} d u_{i} / d x$. For brevity, the dependence on $x$ is not explicitly indicated here and in what follows.

From the kinematical hypothesis (1), the following set of differential equations linking the unknown $\theta, u_{i}$ and $w$ can be obtained.

$$
\begin{aligned}
& E_{1} A_{1} \frac{d^{2} u_{1}}{d x^{2}}+k_{1}\left(u_{2}-u_{1}+e_{1} \cdot \theta\right)=0 \\
& E_{2} A_{2} \frac{d^{2} u_{2}}{d x^{2}}-k_{1}\left(u_{2}-u_{1}+e_{1} \cdot \theta\right)+k_{2}\left(u_{3}-u_{2}+e_{2} \cdot \theta\right)=0 \\
& E_{3} A_{3} \frac{d^{2} u_{3}}{d x^{2}}-k_{2}\left(u_{3}-u_{2}+e_{2} \cdot \theta\right)=0 \\
& G A\left(\frac{d^{2} w}{d x^{2}}-\frac{d \theta}{d x}\right)=q \\
& E I \frac{d^{2} \theta}{d x^{2}}-G A\left(\frac{d w}{d x}-\theta\right)-e_{1} k_{1}\left(u_{2}-u_{1}+e_{1} \cdot \theta\right) \\
& -e_{2} k_{2}\left(u_{3}-u_{2}+e_{2} \cdot \theta\right)=0
\end{aligned}
$$

where $\quad E I=E_{1} I_{1}+E_{2} I_{2}+E_{3} I_{3}, G A=G_{1} A_{1}+G_{2} A_{2}+$ $G_{3} A_{3}$, is the cross-sectional transverse stiffness of the multilayered beam and $q(x)$ is the distributed lateral load acting along the beam direction. The second-order differential system (4) couples the displacements $u_{i}, w$ and rotation angle $\theta$ in the framework of Timoshenko beam theory. By definition, the transverse shear force $V(x)$ is given as

$$
V=G A\left(\theta-\frac{d w}{d x}\right)
$$

such that $q=-\frac{d V}{d x}$, as usual. Note that the fourth equation in (4) comes from the derivative of (5) with respect to $x$, coupling thus the beam curvature $w^{\prime \prime}$ with $\theta^{\prime}$ and $q$. The prime symbol is also used to denote the derivative of a function with respect to $x$.

Next, the differential system (4) can be transformed to a system of ordinary differential equations (ODEs) of the form

$$
\frac{d \boldsymbol{y}}{d x}=\boldsymbol{f}(x, \boldsymbol{y}), \quad 0 \leq x \leq L
$$

where $L$ stands for the beam span. For the Timoshenko theory, one has to consider the $10 \times 1$ column vector $\boldsymbol{y}=\left(\begin{array}{llllllllll}u_{1} & u_{1}^{\prime} & u_{2} & u_{2}^{\prime} & u_{3} & u_{3}^{\prime} & w & w^{\prime} & \theta & \theta^{\prime}\end{array}\right)^{T}$.

From (4), the components of the $10 \times 1$ vector function $f$ are given by

$$
\boldsymbol{f}=\left(\begin{array}{c}
u_{1}^{\prime} \\
-\frac{k_{1}}{E_{1} A_{1}}\left(u_{2}-u_{1}+e_{1} \cdot \theta\right) \\
u_{2}^{\prime} \\
\frac{k_{1}}{E_{2} A_{2}}\left(u_{2}-u_{1}+e_{1} \cdot \theta\right)-\frac{k_{2}}{E_{2} A_{2}}\left(u_{3}-u_{2}+e_{2} \cdot \theta\right) \\
u_{3}^{\prime} \\
\frac{k_{2}}{E_{3} A_{3}}\left(u_{3}-u_{2}+e_{2} \cdot \theta\right) \\
\theta+\frac{V}{G A} \\
\theta^{\prime}+\frac{q}{G A} \\
\theta^{\prime} \\
\frac{1}{E I}\left(G A\left(w^{\prime}-\theta\right)+e_{1} k_{1}\left(u_{2}-u_{1}+e_{1} \cdot \theta\right)\right. \\
\left.+e_{2} k_{2}\left(u_{3}-u_{2}+e_{2} \cdot \theta\right)\right)
\end{array}\right)
$$

The solution of the problem requires the specification of two-point boundary conditions given here in the generic form

$\boldsymbol{g}(\boldsymbol{y}(0), \boldsymbol{y}(L))=\mathbf{0}$

or explicitly,

$w(0))=w(L)=0$,

$w^{\prime \prime}(0)=\theta^{\prime}(0)+V(0) / G A=0$,

$w^{\prime \prime}(L)=\theta^{\prime}(L)+V(L) / G A=0$,

$u_{1}^{\prime}(0)=u_{2}^{\prime}(0)=u_{3}^{\prime}(0)=0$,

$u_{1}(0)+u_{1}(L)=0$,

$u_{2}(0)+u_{2}(L)=0$,

$u_{3}(0)+u_{3}(L)=0$.

The first four boundary conditions in (8) impose that vertical displacements and curvatures are zero at the supports. Axial deformations and stresses are taken to be zero at $x=0$ using the three subsequent conditions. Finally, the remaining conditions lead to opposite values for the axial displacement at the end points, assuming thus that symmetrical loads with respect to mid span prevail here.

For a three-point bending configuration, with load $F$ at $L / 2, q(x)$ is evaluated using the Heaviside function

$$
\begin{aligned}
& H(x)=\left\{\begin{array}{ll}
0 & \text { if } x<0 \\
1 & \text { if } x \geq 0
\end{array}\right. \text { as } \\
& q(x)=q_{0} \cdot\left(H\left(x-\frac{L}{2}+\frac{l_{0}}{2}\right)-H\left(x-\frac{L}{2}-\frac{l_{0}}{2}\right)\right)
\end{aligned}
$$


where $q_{0}=F / l_{0}$ and $l_{0}=L / 100$ is typically considered. Accordingly, the central load is described as a constant load $q_{0}$ acting on a small domain of size $2 l_{0}$, centered at the position $x=L / 2$.

\section{Experimental study}

\subsection{Introduction to the MTSJ geometry}

Based on linear connections for furniture such as dovetail or Japanese nejiri arigata joints, the MTSJ without adhesive bonding or fasteners allow assembly and direct interlocking of two panel edges at their final in-space location.

As a detailed description of this "integrated mechanical attachment" is given by Robeller (2015), the next section briefly introduces the geometrical parameters of the joint. The connection employed here is a one-degree-of-freedom connection (for the purpose of assembly) where tabs are inserted in slots (Fig. 2a). "Multiple" refers to the reiteration of interlocked tab and slot along the common edge of two connected panels. The geometry of the connection defines the relative positioning of the panels and allows a certain degree of load-transfer between them. The assembly of the two panels is directed along a vector of insertion (see below). The tab contact faces of each part belong to the same contact plane after insertion. In general, the insertion vector and the normal to the locking face are obtained by the following sequence (see Fig. 2a).

If $\boldsymbol{n}_{\boldsymbol{0}}$ and $\boldsymbol{n}_{\boldsymbol{1}}$ are the normal of the two considered panels, the frame of the joint $F=\left(\boldsymbol{u}_{1}, \boldsymbol{u}_{2}, \boldsymbol{u}_{3}\right)$ can be calculated as $\boldsymbol{u}_{1}=\boldsymbol{n}_{0} \times \boldsymbol{n}_{1}, \boldsymbol{u}_{2}=\boldsymbol{n}_{0}$ and $\boldsymbol{u}_{3}=\boldsymbol{u}_{1} \times \boldsymbol{u}_{2}$. Then, the line segment representing the intersection of the two panel midplanes can be uniformly divided into $N$ points $\left(X_{i}\right)_{(i=1 . . N)}$, the distance between points is the tab length of the joint $L_{t}$ (Fig. 3). The plane $P_{i}$ is the plane of normal $\boldsymbol{u}_{1}$, and contains the point $X_{i}$. Thus by three successive rotations of this plane and its attached frame as,

$$
\begin{gathered}
\left\{P_{i}, F=\left(\boldsymbol{u}_{\mathbf{1}}, \boldsymbol{u}_{\mathbf{2}}, \boldsymbol{u}_{\mathbf{3}}\right)\right\} \\
R\left(\boldsymbol{u}_{\boldsymbol{1}}, \theta_{1}\right) \downarrow \\
\left\{P_{i}^{\prime}, F^{\prime}=\left(\boldsymbol{u}_{\boldsymbol{1}}^{\prime}=\boldsymbol{u}_{\boldsymbol{1}}, \boldsymbol{u}_{\mathbf{2}}^{\prime}, \boldsymbol{u}_{\mathbf{3}}^{\prime}\right)\right\} \\
R\left(\boldsymbol{u}_{\mathbf{2}}^{\prime}, \theta_{2}\right) \downarrow \\
\left\{P_{i}^{\prime \prime}, F^{\prime \prime}=\left(\boldsymbol{u}_{\boldsymbol{1}}^{\prime \prime}, \boldsymbol{u}_{\mathbf{2}}^{\prime \prime}=\boldsymbol{u}_{\mathbf{2}}^{\prime}, \boldsymbol{u}_{\mathbf{3}}^{\prime \prime}\right)\right\} \\
R\left(\boldsymbol{u}_{\mathbf{3}}^{\prime \prime}, \pm \theta_{3, i}\right) \downarrow \\
\left\{P_{i}^{\prime \prime \prime}, F^{\prime \prime \prime}=\left(\boldsymbol{u}_{\boldsymbol{1}, i}^{\prime \prime \prime}, \boldsymbol{u}_{\mathbf{2}, i}^{\prime \prime}, \boldsymbol{u}_{\mathbf{3}, i}^{\prime \prime \prime}=\boldsymbol{u}_{\mathbf{3}}^{\prime \prime}\right)\right\}
\end{gathered}
$$

the final joint is obtained defining the planes $P_{i}^{\prime \prime \prime}, \boldsymbol{u}_{\boldsymbol{l}, i}^{\prime \prime \prime}$ and $\boldsymbol{u}_{\boldsymbol{3}, i}^{\prime \prime \prime}=\boldsymbol{u}_{\mathbf{3}}^{\prime \prime}$ as the tab contact faces, their normal and the insertion vector, respectively. In the present paper, the $\theta_{1}$, $\theta_{2}$ angles are taken equal to $0^{\circ}$ (Fig. 2b) and the $\theta_{3}$ angle is set successively to $0^{\circ}$ (finger joint), $\pm 15^{\circ}$ (DJ), $\pm 25^{\circ}$ (DJ). Then, four panels are connected with the MTSJ to build a square box beam (Fig. 2c). Note that in this subsection, $\theta_{i}$ and the prime symbol denote the rotation angles and the successive rotated planes and frames, respectively.

\subsection{Description of the samples}

Ten series of box beams with different connection types have been constructed using LVL Kerto-Q panels of 21 $\mathrm{mm}$ thickness with spruce ply (0-90-0-0-0-90-0). The Kerto-Q panels were supplied by the Company Metsäwood. Geometrical and mechanical properties of the experimental specimens are used and gathered for clarity in Table 1. The same values are taken for elastic modulus $E_{1}$, $E_{3}$ and the shear modulus $G_{1}, G_{3}$, corresponding to the values (relative to actions perpendicular to the panel plate) of the VTT certificate provided by Metsäwood. Similarly, $E_{2}, G_{2}$ are the values relative to actions in the panel plate. A common beam length of $2432 \mathrm{~mm}$ for the specimens is considered here.

The ten series (a-j) (see Fig. 3) consist of hollow square beams that are assembled with ten particular sequences of joint elements. Depicted in Fig. 3 is the cross-sectional area of the box beams where the mid-thickness profile corresponds to a square of $99.5 \mathrm{~mm}$ side. Nevertheless, no glue has been added during the assembly process of samples (af) and (i-j).

Three replicates of each sample (Fig. 3) were tested using a three-point bending setup as outlined in Fig. 4 . Samples (a-d) each have a joint geometry with angles $\theta_{1}=0^{\circ}, \theta_{2}=0^{\circ}, \theta_{3}= \pm 15^{\circ}$, namely dovetail joint (DJ15) and tabs ranging from 41 to $486 \mathrm{~mm}$ in length. The slot lengths are equal to the tab lengths for each configuration. The samples $\mathrm{i}$ and $\mathrm{j}$ differ in joint geometry to the previous ones, having $\theta_{3}=0^{\circ}$ (DJ00) and $\theta_{3}= \pm 25^{\circ}$ (DJ25), respectively, while their tab length is identical to that of samples $b$ and $f$ (spacing). Recall that lateral panels have identical joint profiles. The upper and lower panels also present the same joint profile that starts this time with a tab element. Samples e and $\mathrm{f}$ are screwed using Würth ASSY screws of $4 \times 50 \mathrm{~mm}$ with spacings identical to tab lengths of samples $\mathrm{a}$ and $\mathrm{b}$, respectively. Samples $\mathrm{g}$ and $\mathrm{h}$ are bonded by PUR gluing via a horizontal and a vertical joint, respectively. These last two samples serve as reference for rigid behaviour of the tested beams. Finally, a total of 30 beams were loaded until failure (three replicates of each sample).

\subsection{Method}

The test beams of length $2432 \mathrm{~mm}$ are simply supported on steel rollers over a span $\mathrm{L}$ of $2210.5 \mathrm{~mm}$. The actuator 

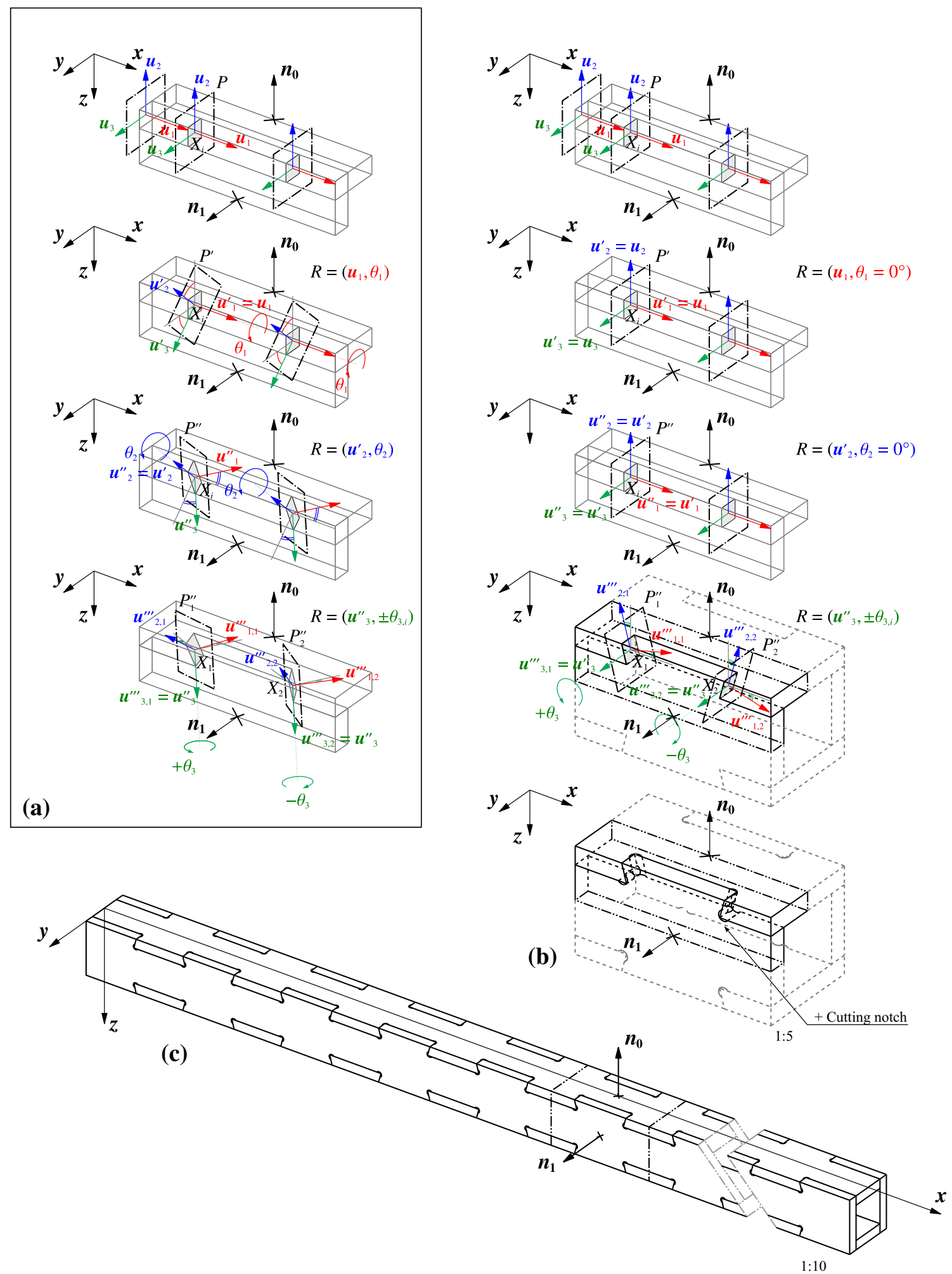

Fig. 2 a General sequence of plane and frame rotations for a MTSJ, b result for a dovetail-type connection with $\theta_{1}, \theta_{2}=0^{\circ}, \mathbf{c}$ boxed beam

motion is controlled automatically, allowing the load to be applied at a constant rate. The load is measured at mid-span using an external load cell placed under the actuator.
Vertical displacements (deflections) are recorded using seven linear variable differential transducers (LVDTs) equally spaced along the beam span (spacing $=368.4 \mathrm{~mm}$ ). 
Sensitivity to tab length

All tab face angles $= \pm 15^{\circ}$
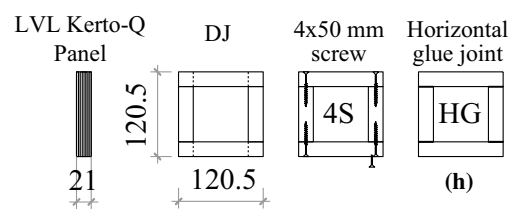

(h)

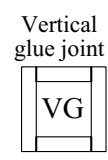

(g)

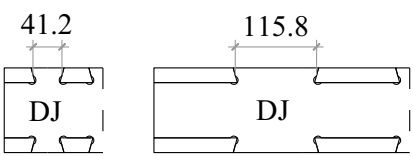

$\rightarrow \square$

(a) 41.2

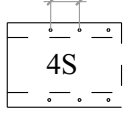

(b) 115.8

$4 \mathrm{~S}$
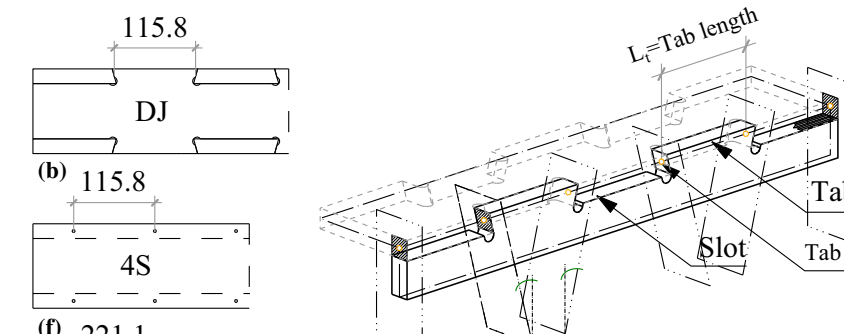

\section{Sensitivity to tab face angle \\ All tab lengths $=115.8 \mathrm{~mm}$}

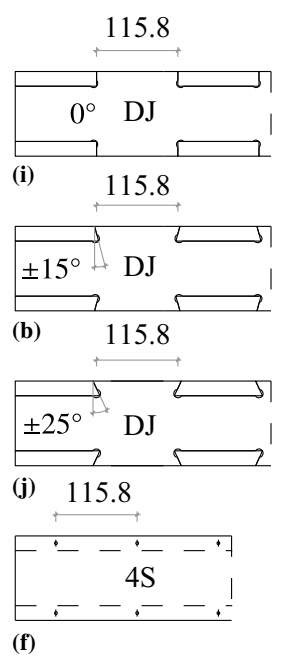

Fig. 3 Sample connection specifications a DJ15 $41 \mathrm{~mm}$, b DJ15 $116 \mathrm{~mm}$, c DJ15 $221 \mathrm{~mm}$, d DJ15 486 mm, e 4 mm-screw $41 \mathrm{~mm}$, f 4 mm-screw $116 \mathrm{~mm}, \mathbf{g}$ vertical glue joint, h horizontal glue joint, i DJ00 $116 \mathrm{~mm}, \mathbf{j}$ DJ25 $116 \mathrm{~mm}$

Table 1 Geometrical and mechanical properties for the 3-layered boxed beams (LVL Kerto-Q from Metsäwood)

\begin{tabular}{llll}
\hline & Layer 1 & Layer 2 & Layer 3 \\
\hline Height $h_{i}(\mathrm{~mm})$ & 21 & 78.5 & 21 \\
Width $b_{i}(\mathrm{~mm})$ & 120.5 & 42 & 120.5 \\
Elastic modulus $E_{i}\left(\mathrm{~N} / \mathrm{mm}^{2}\right)$ & 10,000 & 10,000 & 10,000 \\
Shear modulus $G_{i}\left(\mathrm{~N} / \mathrm{mm}^{2}\right)$ & 60 & 600 & 60 \\
\hline
\end{tabular}

Measurements are performed at the middle of the beam's upper panel, so that transducers (d0) and (d6) are positioned at the support locations and at the actuator (d3), which is placed at mid-span (Fig. 4). Experimental results are given in the next section.

\subsection{Experimental results}

During the experiment, the box beam specimens in each series have been exposed to a simple quasi static loading cycle until failure of the beams.

\subsubsection{Sensitivity to tab length}

In Fig. 5, deflection profiles at a maximal load of $3.0 \mathrm{kN}$ are compared for the $(a-h)$ series of tests; DJ15 type with various tab lengths. For each curve, plot symbols represent data points provided by the transducers equally spaced along the beam length (see Fig. 4). In fact each
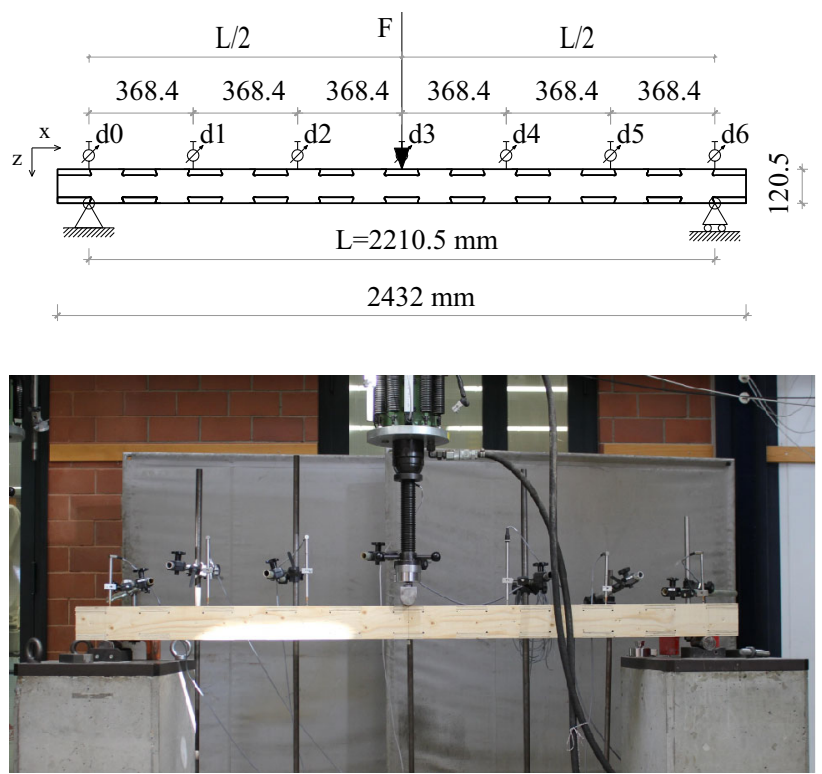

Fig. 4 Three-point bending tests with LVDT locations

measurement point is obtained by averaging all the measurements taken from the replicates in a series. For comparison, the curve representing a rigid beam model is included in Fig. 5.

Practically, the system (6) subjected to the two-point boundary value conditions (7) can be integrated numerically using the so-called $b v p 4 c$ routine of the Matlab software (Shampine et al. 2000). This routine implements 


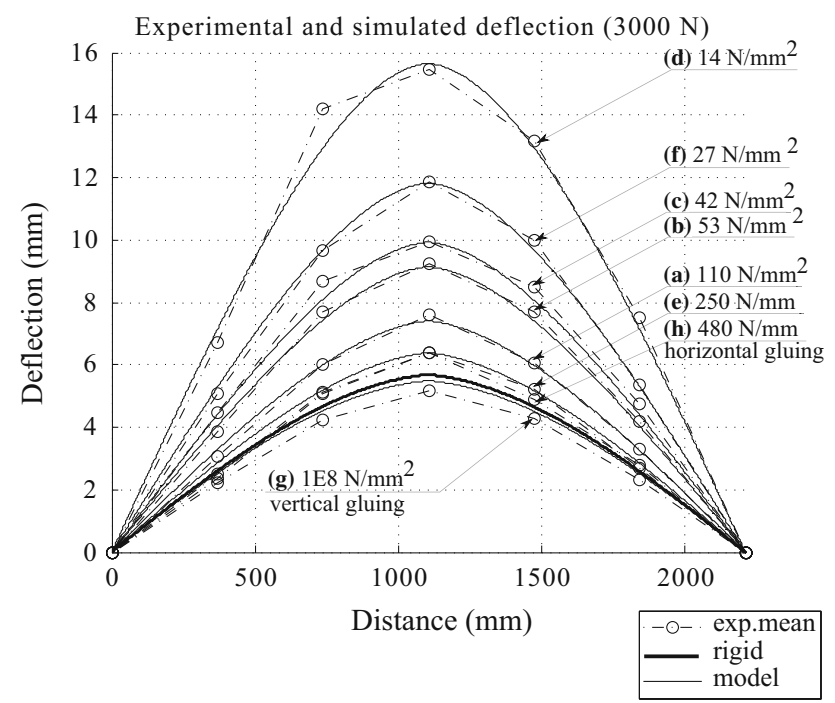

Fig. 5 Deflection curves of the tested beams for $\mathrm{F}=3000 \mathrm{~N}$, average of three runs per specimens $a$ DJ15-41 mm, $b$ DJ15-116 mm, $c$ DJ15$221 \mathrm{~mm}, d$ DJ15-486 mm, e $4 \mathrm{~mm}$-screw $41 \mathrm{~mm}, f 4 \mathrm{~mm}$-screw 116 $\mathrm{mm}, g$ vertical glue joint, $h$ horizontal glue joint

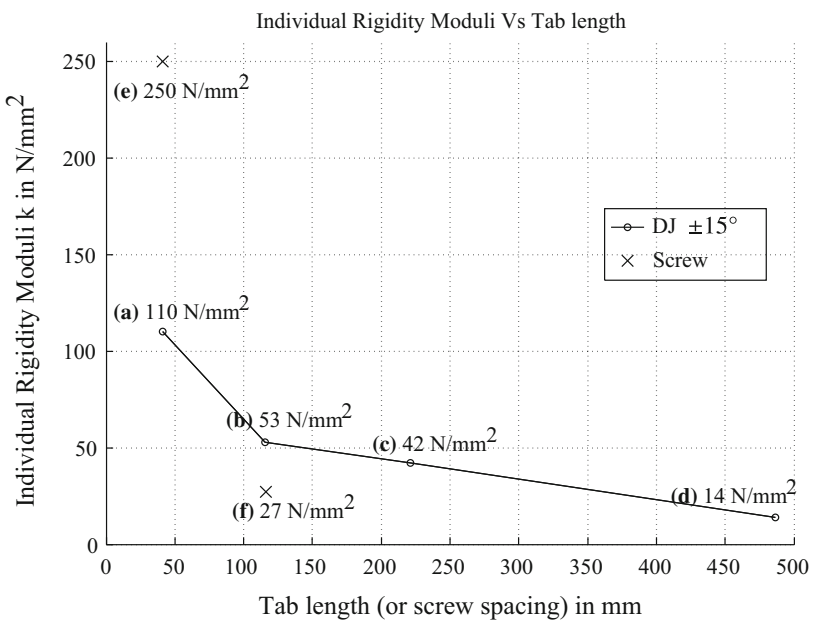

Fig. 6 Individual rigidity modulus vs tab length or screw spacing, $a$ DJ15-41 mm, $b$ DJ15-116 mm, $c$ DJ15-221 mm, $d$ DJ15-486 mm, e $4 \mathrm{~mm}$-screw $41 \mathrm{~mm}, f 4 \mathrm{~mm}$-screw $116 \mathrm{~mm}$

an effective collocation method for the resolution of practical boundary value problems. The solution $\boldsymbol{y}_{\text {sol }}$ is a continuous function that is computed by discretizing the domain on $\mathrm{K}$ subintervals $\left[x_{k}, x_{k+1}\right]$ such as $0=x_{1}<x_{2}<\ldots$ $<x_{K}=L$. Over each subinterval, $\boldsymbol{y}_{\text {sol }}$ is approximated by a cubic polynomial function and satisfies the differential equations (4) at both ends and the midpoint of the subinterval. In the present case, accurate results are obtained by taking $K=1201$ and constant step sizes of $1.84 \mathrm{~mm}$. This value of $K$ guarantees a good execution time without diverging more than $1.2 \%$ from a $K$-value ten times higher. Next, it is worth noting that the rigidity moduli $k_{1}$ and $k_{2}$ appear as parameters in the model that will be determined from the experimental deflections given in Fig. 5.

Semi-rigid values for each connection type are obtained by assuming the same value $k$ for the two interface rigidities, i.e. $k_{1}=k_{2}=k$. In each case, this value is determined iteratively by matching the experimental deflections to the simulated ones. The final $k$-value is fixed when the deviation between the experimental and numerical points at $x=\{0, L / 6, L / 3, L / 2,2 L / 3,5 L / 6, L\}$ is minimum. The amplitude of the deviation is sufficiently low for attesting of the overall elastic behavior of the beams. Experimental and simulated deflections are plotted with dotted and solid lines respectively.

The force varies linearly with the displacement during the loading step and a maximum value of about $16 \mathrm{~mm}$ [(d), $486 \mathrm{~mm}$ tab length] is recorded with LVDT (d3) at $3 \mathrm{kN}$.

The effect of shear was included in the models (about $10 \%$ of additional deformation for this beam geometry). As expected the deflection curves of the glued beam, the rigid model and the numerical model with rigidity of $10^{8}$ $\mathrm{N} / \mathrm{mm}^{2}$ match rather well (see Fig. 5). In Fig. 6, the rigidity coefficient is represented as a function of the tab length of the MTSJ (or spacing for screws). The screw connection is indicated by a cross and DJ15 is indicated by a circle. The ratio of the stiffness $\left(k_{D J 15} / k_{\text {screw }}\right)$ is higher when increasing screw spacing/tab length. It goes from $k_{(\mathbf{a})} / k_{(\mathbf{e})}=0.44$ for $41 \mathrm{~mm}$ to $k_{(\mathbf{b})} / k_{(\mathbf{f})}=1.96$ for $116 \mathrm{~mm}$. The DJ15-connected beam shows a better rigidity when reducing the number of tab elements than the screwed beam when reducing the number of screws (236 elements to 84 elements). Below $116 \mathrm{~mm}$ [samples (c) and (d)], DJ15 does not offer adequate performance as low stiffness is accommodated by a low ultimate strength (see Table 2) due to the shear force being distributed on only a few contact surfaces.

To complete the semi-rigid analysis, the different joint configurations were compared for a maximum beam deflection corresponding to $1 / 200^{\text {th }}$ of the span $L$. The 486 mm-DJ15 connected beam is out of the considered elastic range $\left(0.1-0.4 F_{\max }\right)$. The $116 \mathrm{~mm}-\mathrm{DJ} 15$ connection acts mechanically better than a $116 \mathrm{~mm}$-spacing screwed joint with a load of $3.5 \mathrm{kN}$ as against $2.79 \mathrm{kN}$ ( $25 \%$ more). Within the elastic range, the $41 \mathrm{~mm}$-spacing screwed beam and the $41 \mathrm{~mm}$-DJ connected beam show no more than $7 \%$ of difference between their centre point loads of 4.52 and $4.22 \mathrm{kN}$, respectively. 
Table 2 Deflection of beam samples at $1 / 200$ th of span

\begin{tabular}{lllllll}
\hline Rep & $\begin{array}{l}\text { Deflection (1/200) } \\
\mathrm{mm}\end{array}$ & $\begin{array}{l}\mathrm{F}(1 / 200) \\
\mathrm{kN}\end{array}$ & $\begin{array}{l}0.1 \text { Fmax } \\
\mathrm{kN}\end{array}$ & $\begin{array}{l}0.4 \text { Fmax } \\
\mathrm{kN}\end{array}$ & $\begin{array}{l}\text { Fmax } \\
\mathrm{kN}\end{array}$ & $\begin{array}{l}\text { Deflection max } \\
\mathrm{mm}\end{array}$ \\
\hline (d) DJ15-486 & - & 2.19 & 0.52 & 2.08 & 5.19 & 35.24 \\
(f) S4 mm-116 & - & 2.79 & 0.77 & 3.07 & 7.67 & 71.06 \\
(i) DJ00-116 & - & 2.78 & 1.16 & 4.65 & 11.63 & 50.53 \\
(c) DJ15-221 & - & 3.19 & 1.05 & 4.19 & 10.48 & 52.55 \\
(b) DJ15-116 & 11.05 & 3.50 & 1.14 & 4.55 & 11.37 & 43.80 \\
(a) DJ15-41 & - & 4.22 & 1.22 & 4.89 & 12.23 & 41.65 \\
(e) S4 mm-41 & - & 4.52 & 1.20 & 4.80 & 12.01 & 62.11 \\
(h) H-glued & - & 4.92 & 1.29 & 5.17 & 12.92 & 40.30 \\
(g) V-glued & - & 6.32 & 1.83 & 7.31 & 18.28 & 36.42 \\
\hline
\end{tabular}

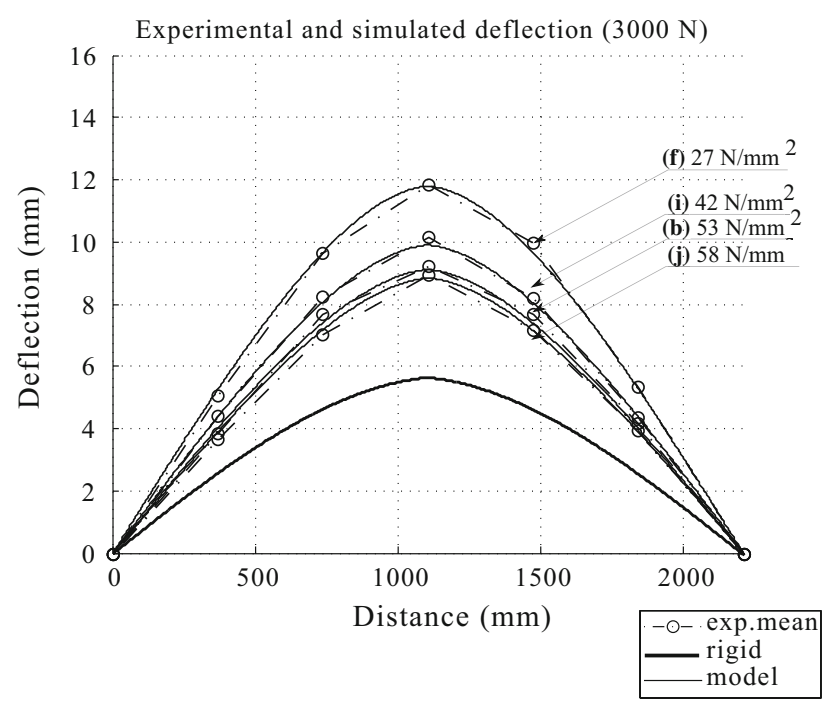

Fig. 7 Deflection curves of the tested beams for $\mathrm{F}=3000 \mathrm{~N}$, average of three runs per specimen $i$ DJ00-116 mm, $b$ DJ15-116 mm, $j$ DJ25$116 \mathrm{~mm}, f 4 \mathrm{~mm}$-screw $116 \mathrm{~mm}$

\subsubsection{Sensitivity to tab contact face angle}

Figure 7 shows deflection profiles at a maximal load of 3.0 $\mathrm{kN}$ for the samples i-DJ00, b-DJ15, j-DJ25 and f-4 mm screw. The tab length (or screw spacing) is set to $116 \mathrm{~mm}$ for each sample.

The geometry of the i-DJ00 is similar to the one of a traditional finger joint. Consequently, the upper and lower panels are only blocked from a vertical displacement by a possible friction between the contact faces of the tabs and the slots. As soon as the tab face angle increases to $15^{\circ}$ and $25^{\circ}$, the rigidity grows by 26 and $38 \%$, respectively. A difference of less than $10 \%$ between b-DJ15 and j-DJ25 cannot be considered as significant. Using larger angles, like $25^{\circ}$ for instance, induces inclination of the contact normal that increases the transverse components of stresses. The loads, perpendicular to the panel grain, are higher. So the presence of cross layers in the panel plies takes all

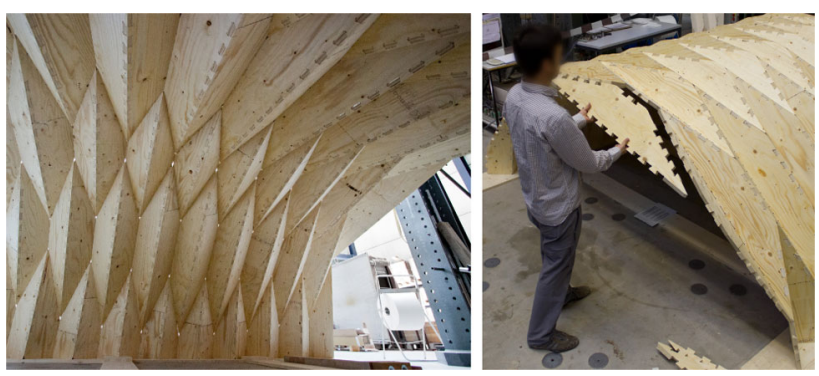

Fig. 8 The MTSJ joints have already been successfully applied to more complex assemblies, where the joint angles $\theta_{1}, \theta_{2}$ and $\theta_{3}$ are not zero (Robeller et al. 2014).

its importance. Simek and Sebera (2010) mention that previous studies have shown an optimal angle between $10^{\circ}$ and $14^{\circ}$.

\section{Conclusion}

This paper focuses not directly on geometrically complex assemblies, but on the development and improvement of joints that allow for the design and fabrication of those. From the architectural point of view, the main priorities are considered to be geometric variability, efficient design, fabrication and assembly, as well as a visual appearance that must not be hidden. However, in order to develop such connections for structural building components, the mechanical behavior must be analyzed thoroughly. The specimens have therefore been chosen with a simple geometry for these first experiments and models, although the connections have already been successfully applied to various other assemblies, as shown in Fig. 8 (Robeller et al. 2014).

The experimental results presented in this paper allow for a comparison with other state-of-the-art joints. The MTSJ provides a highly feasible alternative to the traditional screwed connection. Compared to a reasonable 
screw spacing (116 mm, 84 screws), a box beam connected with MTSJ-dovetail type gets $96 \%$ of rigidity with a much lower assembly time. One should note that a screwed assembly would not comply with the desired standard (EN 1995) when used in conjunction with the $21 \mathrm{~mm}$ Kerto-Q panels employed for the study. Typically, a minimal thickness of $40 \mathrm{~mm}$ (at least ten times the screw diameter) is required for connecting edgewise panels with $4 \mathrm{~mm}$ screw in the absence of dedicated testing. The experimental results are generally encouraging; they figure out that the MTSJ joints behave efficiently as a semi-rigid connection. Within the field of complex geometry, IBOIS has initiated teamwork to design different timber folded-plate structures connected with MTSJ joint. Modeling the semi-rigid behavior of the connection is of main importance for their global structural analysis. The behavior of these joints will be further explored under other loading cases (e.g. moment about edges of short-plate elements).

\section{References}

EN 1995-1-1 (2004) Eurocode 5: design of timber structures-part 1-1: general-common rules and rules for buildings. European Committee for Standardization, Brussels

Engström DB (1992-1999) Evaluation of connections by the use of semi-rigidity. In: Haller $\mathrm{P}$ (ed) Semi-rigid timber jointsstructural behaviour, modelling and new technologies. Semirigid behaviour of civil engineering structural connections. COST C1. Final report of working group "Timber Joints". Technische Universität Dresden

European Technical Approval ETA-11/0190 (2013) self-tapping screws for use in timber constructions. Deutsches Institut für Bautechnik, p16

Goodman JR, Popov EP (1968) Layered beam systems withinterlayer slip. J Struct Div 94(11):2535-2547. Proc Am Soc CivilEng
Graubner W (1987) Comparison of Japanese and European joining solutions (in German), 2nd edn. Deutsche Verlags-Anstalt, Stuttgart

Krawczyk P, Frey F, Zielinsky AP (2007) Large deflections of laminated beams with interlayer slips part 1: model development. Eng Comput 24(1):17-32

Kreuzinger H (1995) Mechanically jointed beams and columns, STEP 1, timber engineering, B11/1-8. Centrum Hout, The Netherlands

Möhler K (1956) On the load-bearing behaviour of beams and compressive members with composite cross-sections and semirigid connectors (in German). Habilitation, Technische Universität Karlsruhe, Germany

Natterer J, Sandoz JL, Rey M (2004) Treaty of civil engineering at EPFL : wood construction, materials, technology and design (in French). PPUR, vol 13, 2nd edn

Newmark NM, Siess CP, Viest IM (1951) Tests and analysis of composite beams with incomplete interaction, 9(1):75-92. Proc Soc Exp Stress Anal

Robeller C, Stitic A, Mayencourt P, Weinand Y (2014) Interlocking folded plate, integrated mechanical attachment for structural timber panels, advances in architectural geometry 2014, University College London, United Kingdom, September 18-19, 2014, Advances in Architectural Geometry 4

Robeller C (2015) Integral mechanical attachment for timber folded plate structures. Thesis, Swiss Federal Institute for Technology Lausanne (EPFL)

Schelling W (1968) The calculation of composite beams with semi rigid connectors in timber construction (in German). Dissertation, TH Karlsruhe

Shampine LF, Reichelt MW, Kierzenka J, (2000) Solving boundary value problems for ordinary differential equations in MATLAB with bvp4c. http://www.mathworks.com/bvp_tutorial

Simek M, Sebera V (2010) Finite element analysis of dovetail joint made with the use of cnc technology. Acta Universitatis Agriculturae Et Silviculturae Mendelianae Brunensis, vol LVIII, N5, pp 321-328

Sousa Jr JBM, Da Silva AR (2010) Analytical and numerical analysis of multilayered beams with interlayer slip. Eng Struct $32: 1671-1680$ 